been definitely excluded from this result by previous assumption that $x<n$.

For other values of $d$ the expressions in (5) do not simplify, and we have to determine $x$ from the general conditions

$n=a x+c, c<x,(a \neq 0, a-c>(d-1)(x+1)$ and $\leqq d(x+1))$.

It may be verified by actual trial that the solutions of (1) and (2) may in this way be effected with a degree of readiness which will make the method serviceable in a large number of cases.

Alabama Presbyterian College, anniston, Alabama.

\title{
THE INNER FORCE OF A MOVING ELECTRON.
}

\author{
BY DR, F. R. SHARPE.
}

(Read before the American Mathematical Society, February 29, 1908.)

1. Introduction. - Sommerfeld ${ }^{*}$ has given a general method of determining the force which an electron exerts on itself when its motion is known. Schott $\dagger$ has applied the retarded potential to the same problem. In the present paper the known vector expression $\$$ for the force with which a moving point charge acts on another point charge is used to determine the action between any two elements of the electron. A double integration over the volume of the electron gives the inner force of the electron. The Abraham-Sommerfeld expressions for the longitudinal mass and Abraham's value for the transverse mass are thus very simply determined and the limitations on the solutions are made manifest.

2. The Force Between Two Moving Point Charges. - The electron is assumed to be a uniformly charged solid sphere which is moving in a straight line without rotation. Take the $x$-axis in the direction of motion. Measure the time from the instant at which the force is to be determined and choose the velocity of light as the unit of velocity. Let $\left(x_{0}, y, z\right)$ be the coordinates of the point charge $d e_{2}$ relative to the point charge $d e_{1}$. The distance moved by the electron in time $t$ is

* Göttinger Nachrichten, 1904.

†Ann. der. Physik, 1908, No. 1.

$\ddagger$ Abraham : Theorie der Elektrizität, vol. II, p. 98. 


$$
v_{0} t+\frac{1}{2} \dot{v}_{0} t^{2}+\frac{1}{6} \ddot{v}_{0} t^{3}+\cdots,
$$

where $v_{0}$ is its velocity at time 0 . The point charge $d e_{1}$ will therefore act on $d e_{2}$ at the time $-\tau$ which is determined so as to satisfy

$$
\tau^{2}=y^{2}+z^{2}+\left(x_{0}+v_{0} \tau-\frac{1}{2} \dot{v}_{0} \tau^{2}+\cdots\right)^{2} .
$$

The velocity of $d e_{1}$ at this time is

$$
v=v_{0}-\dot{v}_{0} \tau+\frac{1}{2} \ddot{v}_{0} \tau^{2}-\cdots,
$$

and its acceleration is

$$
\dot{v}=\dot{v}_{0}-\ddot{v}_{0} \tau+\cdots
$$

If the velocity of $d e_{1}$ had remained constant and equal to $v$ from the time $-\tau$, its coordinates at time 0 would have been $(x, y, z)$, where

or

$$
x=x_{0}+v_{0} \tau-\frac{1}{2} \ddot{v}_{0} \tau^{2}+\cdots-\tau\left(v_{0}-\dot{v}_{0} \tau+\cdots\right)
$$

$$
x=x_{0}+\frac{1}{2} \dot{v}_{0} \tau^{2}+\cdots
$$

The $x$ component of the force of $d e_{1}$ on $d e_{2}$ can be reduced from its vector form to

$$
\frac{x\left(1-v^{2}\right)-\dot{v}\left(y^{2}+z^{2}\right)}{\left\{x^{2}+\left(1-v^{2}\right)\left(y^{2}+z^{2}\right)\right\}^{\frac{3}{2}}} d e_{1} d e_{2} .
$$

The effect of $d e_{2}$ on $d e_{1}$ is obtained by changing the sign of $x_{0}$.

3. Uniform Motion. - In this case $v$ is $v_{0}$ and $x$ is $x_{0}$, so that (5) becomes

$$
\frac{x_{0}\left(1-v_{0}^{2}\right)}{\left\{x_{0}^{2}+\left(1-v_{0}^{2}\right)\left(y^{2}+z^{2}\right)\right\}^{\frac{3}{2}}} d e_{1} d e_{2} .
$$

Hence the mutual actions of $d e_{1}$ and $d e_{2}$ are equal and opposite and the inner force is zero.

4. Slow Uniform Acceleration.-Here $\dot{v}$ is $\dot{v}_{0}$, and the unbalanced part of the force of $d e_{1}$ on $d e_{2}$ is

$$
-\frac{\dot{v}_{0}\left(y^{2}+z^{2}\right) d e_{1} \cdot d e_{2}}{\left\{x_{0}^{2}+\left(1-v_{0}^{2}\right)\left(y^{2}+z^{2}\right)\right\}^{\frac{3}{2}}},
$$

which may be written 


$$
-\frac{\dot{v}_{0} \partial}{v_{0} \partial v_{0}}\left[\frac{d e_{1} d e_{2}}{\left\{x_{0}^{2}+\left(1-v_{0}^{2}\right)\left(y^{2}+z^{2}\right)\right\}^{\frac{1}{2}}}\right] .
$$

The inner force of the electron is twice the sum of this expression for every pair of elements of the electron. Let the coordinates of $d e_{1}$ and $d e_{2}$ referred to the center of the electron be $\left(x_{1}, y_{1}, z_{1}\right)$ and $\left(x_{2}, y_{2}, z_{2}\right)$. Then

$$
x_{0}=x_{2}-x_{1}, \quad y=y_{2}-y_{1}, \quad \text { and } z=z_{2}-z_{1} .
$$

If we now make the transformation

$$
x=\sqrt{1-v_{0}^{2}} \cdot x^{\prime}, \quad \rho=\frac{\rho^{\prime}}{\sqrt{1-v_{0}^{2}}},
$$

where $\rho$ is the volume density of the charge $e$ of the electron, the charge is unaltered, but the spherical electron of radius $a$ is transformed into the prolate spheroid

$$
x^{\prime 2}\left(1-v_{0}^{2}\right)+y^{2}+z^{2}=a^{2} .
$$

The expression which we have to integrate is therefore $2 / \sqrt{\left(1-v_{0}^{2}\right)}$ of the self potential of this spheroid and has the value $*$

$$
\frac{6}{5} \cdot \frac{e^{2}}{2 a \dot{v}_{0}} \log \left(\frac{1+v_{0}}{1-v_{0}}\right) .
$$

Hence the inner force of the electron is

that is,

$$
-\frac{6}{5} \cdot \frac{e^{2} \dot{v}_{0}}{2 a} \cdot \frac{\partial}{v_{0} \partial v_{0}}\left\{\frac{1}{v_{0}} \log \left(\frac{1+v_{0}}{1-v_{0}}\right)\right\},
$$

$$
-\dot{v}_{0} \cdot \frac{6}{5} \frac{e^{2}}{2 \alpha v_{0}^{2}}\left(-\frac{1}{v_{0}} \log \frac{1+v_{0}}{1-v_{0}}+\frac{2}{1-v_{0}^{2}}\right) \text {. }
$$

The coefficient of $-\dot{v}_{0}$ is Abraham's expression for the longitudinal mass in the case of quasi-stationary motion.

5. Variable Acceleration. - In the previous case the changes in the velocity and acceleration were neglected. If we retain the first power of $\tau$, we have from (2) and (3)

* Abraham, loc. cit., p. 179.

$$
v=v_{0}-\dot{v}_{0} \tau, \quad \dot{v}=\dot{v}_{0}-\ddot{v}_{0} \tau,
$$


and from (1)

$$
\tau=\frac{v_{0} x+\sqrt{x_{0}^{2}+\left(1-v_{0}^{2}\right)\left(y^{2}+z^{2}\right)}}{1-v_{0}^{2}} .
$$

Substituting in (5), we find the additional unbalanced terms $\frac{\ddot{v}_{0}\left(y^{2}+z^{2}\right) d e_{1} d e_{2}}{\left(1-v_{0}^{2}\right)\left\{x_{0}^{2}+\left(1-v_{0}^{2}\right)\left(y^{2}+z^{2}\right\}\right.}+\frac{3 \dot{v}_{0}^{2} v_{0}\left(y^{2}+z^{2}\right)^{2} d e_{1} d e_{2}}{\left(1-v_{0}^{2}\right)\left\{x_{0}^{2}+\left(1-v_{0}^{2}\right)\left(y^{2}+z^{2}\right)\right\}^{2}}$. Denoting by $\theta$ the angle which the line joining $d e_{1}$ to $d e_{2}$ makes with the $x$ axis, we have

$$
\frac{\ddot{v}_{0}}{1-v_{0}^{2}} \cdot \frac{\sin ^{2} \theta d e_{1} d e_{2}}{1-v_{0}^{2} \sin ^{2} \theta}+\frac{3 \dot{v}_{0}^{2}}{2\left(1-v_{0}^{2}\right)} \frac{\partial}{\partial v_{0}}\left(\frac{\sin ^{2} \theta d e_{1} d e_{2}}{1-v_{0}^{2} \sin ^{2} \theta}\right) .
$$

Consider

$$
\iint \frac{\sin ^{2} \theta d e_{1} d e_{2}}{1-v_{0}^{2} \sin ^{2} \theta}
$$

since it is independent of the length of the line joining $d e_{1}$ to $d e_{2}$, its value is

$$
\frac{e^{2}}{4 \pi} \int \frac{\sin ^{2} \theta}{1-v_{0}^{2} \sin ^{2} \theta} d w,
$$

where $d w$ is the element $2 \pi \sin \theta d \theta$ of the complete solid angle $4 \pi$. Integrating from 0 to $\pi$, we find the value of the additional terms to be

$$
\begin{aligned}
\frac{\ddot{v}_{0} e^{2}}{1-v_{0}^{2}}(- & \left.\frac{1}{v_{0}^{2}}+\frac{1}{v_{0}^{3} \sqrt{1-v_{0}^{2}}} \tan ^{-1} \frac{v_{0}}{\sqrt{1-v_{0}^{2}}}\right) \\
& +\frac{3 \dot{v}_{0}^{2} e^{2}}{2\left(1-v_{0}^{2}\right)} \frac{\partial}{\partial v_{0}}\left(-\frac{1}{v_{0}^{2}}+\frac{1}{v_{0}^{3} \sqrt{1-v_{0}^{2}}} \tan ^{-1} \frac{v_{0}}{\sqrt{1-v_{0}^{2}}}\right) .
\end{aligned}
$$

This result differs somewhat from the expression found by Schott and Abraham.

6. Transverse Acceleration. - When the velocity is constant but there is a small transverse acceleration $f$, we may choose the $y$ axis in the direction of $f$. The vector expression for the $y$ component of the unbalanced force may be reduced to

$$
-f \frac{x_{0}^{2}+\left(1-v_{0}^{2}\right) z^{2}}{\left\{x_{0}^{2}+\left(1-v_{0}^{2}\right)\left(y^{2}+z^{2}\right)\right\}^{\frac{3}{2}}} d e_{1} d e_{2},
$$


that is,

$-f d e_{1} d e_{2}\left[\frac{1}{\left\{x_{0}^{2}+\left(1-v_{0}^{2}\right)\left(y^{2}+z^{2}\right)\right\}^{\frac{1}{2}}}-\frac{\left(1-v_{0}^{2}\right) y^{2}}{\left\{x_{0}^{2}+\left(1-v_{0}^{2}\right)\left(y^{2}+z^{2}\right)\right\}^{\frac{3}{2}}}\right]$.

Integrating as in the previous cases, we have

or

$$
-f \cdot \frac{6}{5} \cdot \frac{e^{2}}{2 a}\left\{\frac{1}{v_{0}} \log \frac{1+v_{0}}{1-v_{0}}-\frac{\left(1-v_{0}^{2}\right) \partial}{2 v_{0} \partial v_{0}}\left(\frac{1}{v_{0}} \log \frac{1+v_{0}}{1-v_{0}}\right)\right\}
$$

$$
-f \cdot \frac{6}{5} \cdot \frac{e^{2}}{2 a v_{0}^{2}}\left\{\frac{1+v_{0}^{2}}{2 v_{0}} \log \frac{1+v_{0}}{1-v_{0}}-1\right\},
$$

which gives Abraham's expression for the transverse mass.

CoRNELL UNIVERSITY, March, 1908.

\section{THE RECENTLY DISCOVERED MANUSCRIPT OF ARCHIMEDES.}

Professor J. L. Heiberg has published two important accounts of his recent discovery of a new manuscript of Archimedes, both of which are of great interest to mathematicians. The first of these accounts is printed in volume 42 of Hermes. It contains the Greek text of a lost treatise of Archimedes, which is recovered nearly complete in the newly found manuscript. A German translation of the Greek text, and an interesting commentary by Zeuthen, is printed by Heiberg in the Bibliotheca Mathematica, volume 7, page 321.

Professor Heiberg's critical study of Archimedes has extended over a period of more than thirty years. His dissertation, "Quæstiones Archimedeæ" (Copenhagen, 1879), is constantly referred to by students of Archimedes, both on account of its scholarly critique of Archimedes's work, and on account of the innumerable references to Archimedean literature which are there brought together for the first time. In 1880-1881 Heiberg published the definitive edition of Archimedes, with which his name is usually associated. It was while at work upon a second edition of this book that Heiberg's attention was directed to a palimpsest manuscript of mathematical content recently catalogued in a cloister at Constantinople. Failing in an attempt to have the manuscript sent to him at Copen- 